\title{
2. Beobachtungen über den Effekt des elektrischen Feldes auf Spektrallinien. II. Längseffekt; ${ }^{1}$ ) von J. Stark und G. Wendt.
}

§ 1. Problem. An anderer Stelle ${ }^{2}$ ) hat der eine von uns in einer ersten Mitteilung den Quereffekt (Transversaleffekt) des elektrischen Feldes auf Serienlinien des Wasserstoffes und Heliums beschrieben. Auf Grund der Resultate über diesen Quereffekt ließen sich für den Längseffekt des elektrischen Feldes auf Spektrallinien folgende Verhältnisse erwarten. Diejenigen Komponenten einer elektrisch zerlegten Linie, welche im Quereffekt elektrisch senkrecht zu den elektrischen Kraftlinien geradlinig schwingen, erscheinen allein im Längseffekt und sind in diesem unpolarisiert.

Wenn diese Vorhersage auch viel Wahrscheinlichkeit für sich hatte, so erschien es doch notwendig, sie experimentell zu prüfen. Denn es durfte nicht ohne weiteres als gänzlich ausgeschlossen gelten, daB in einigen Fällen gewisse Komponenten einer zerlegten Linie im Längseffekt zirkular polarigiert sind. Unterliegt nämlich einerseits das Atom einer Serienlinie etwa als Dipol einer orientierenden Kraft im elektrischen Feld, wie es wahrscheinlich ist, hat also eine Atomachse das Bestreben sich parallel der Achse des elektrischen Feldes zu stellen, und vermag dasjenige Elektron in ihm, welches die Serienlinie emittiert, infolge eines $Z_{\text {wanges der Atomstruktur nur in einer }}$ Richtung um jene Atomachse zu rotieren, so muB die von diesem Elektron in der Feldachse emittierte Spektrallinie wenigstens zum Teil zirkular polarisiert sein.

Nun sei als Resultat unserer Untersuchung vorweg mitgeteilt, daB die Komponenten einer Serienlinie im Längseffekt wenigstens für Wasserstoff und Helium unpolarisiert sind.

1) Aus den Nachrichten der K. Ges. d. Wiss. zu Göttingen, Sitzung am 20. Dezember 1913.

2) J. Stark, Ber. d. Berl. Akad. 47. p. 932. 1913; vorausgehende Abhandlung I. 
Hieraus ergibt sich gemäB vorstehender Überlegung die Folgerung, daB die Elektronen, welche die Zentra der Serienemission sind, bei diesen Elementen um eine Atomachse parallel der Feldachse sowohl in der einen als in der entgegengesetzten Richtung zu rotieren vermögen.

Der Längseffekt des elektrischen Feldes auf Serienlinien ist demnach von dem Zeemaneffekt in bemerkenswerter Weise verschieden: während in diesem die Komponenten einer zerlegten Serienlinie rechts und links zirkular polarisiert sind, erweisen sie sich im Längseffekt des elektrischen Feldes unpolarisiert. Und wie ebenfalls gleich hier bemerkt sei, ist dies Resultat der Beobachtung, Fehlen der Polarisation im Längseffekt des elektrischen Feldes, in Übereinstimmung mit der von $\mathrm{W}$. Voigt ${ }^{1}$ ) gegebenen Theorie der Zerlegung von Spektrallinien durch ein elektrisches Feld.

Die experimentelle Untersuchung des Längseffektes des elektrischen Feldes auf Serienlinien hat auch für die Auffindung des neuen Effektes an gewissen Stellen der Sonnenatmosphäre Bedeutung. Es scheint nämlich, daB dort ähnlich wie in der Erdatmosphäre die elektrischen Kraftlinien überwiegend radiale Richtung haben. Ist dies der Fall, so wird ein Beobachter, der in ein radiales elektrisches Feld auf der Mitte der Sonnenscheibe schaut, den Längseffekt und, wenn er in ein radiales Feld am Rande der Sonnenscheibe blickt, den Quereffekt beobachten.

§ 2. Methoden. In der erwähnten ersten Mitteilung hat der eine von uns folgendes methodische Prinzip zur Herstellung eines starken elektrischen Feldes in einem leuchtenden Gas angegeben: die Lichtemission in einem verdünnten Gas wird durch Kanalstrahlen angeregt, auf das so außerhalb eines Glimmstromes leuchtende Gas in engem Raume wird das elektrische Feld einer unselbständigen Strömung gelegt. Zum Zweck der Beobachtung des Quereffektes des elektrischen Feldes wurde das vorstehende Prinzip so ausgeführt, daB die Achse der Licht emittierenden Kanalstrahlen in die Achse des elektrischen Feldes gelegt wurde; die Sehrichtung stand also gleichzeitig senkrecht auf der Achse des Feldes und auf der Bewegungsrichtung der

1) W. Voig t, Ann. d. Phys. 4. p. 197. 1901. 
Kanalstrahlen. Wollte man bei dieser Anordnung den Längseffekt des elektrischen Feldes beobachten, so würde dies durch folgende Umstände sehr erschwert; einmal würde neben dem Licht aus dem starken elektrischen Feld noch intensives Licht durch die Löcher in der Kathode aus dem Raum vor der Kathode (erste Kathodenschicht) in die Sehrichtung gelangen, zweitens wïrde der Effekt des elektrischen Feldes durch den Dopplereffekt der leuchtenden Kanalstrahlenteilchen gestört werden.

Um den vorstehenden Schwierigkeiten aus dem Wege zu gehen, haben wir das obige methodische Prinzip in folgender Ausfübrung bei unserer Untersuchung des Längseffektes zur Anwendung gebracht. Wir haben nämlich das elektrische Feld senkrecht zur Achse der Kanalstrahlen gestellt und in der Achse des Feldes parallel zur Hinterfläche der Glimmstromkathode durch Löcher in der Anode des Spannungsfeldes hindurch die Serienemission in diesem beobachtet. Die Einzelheiten der von uns gewählten Versuchsanordnung waren folgende.

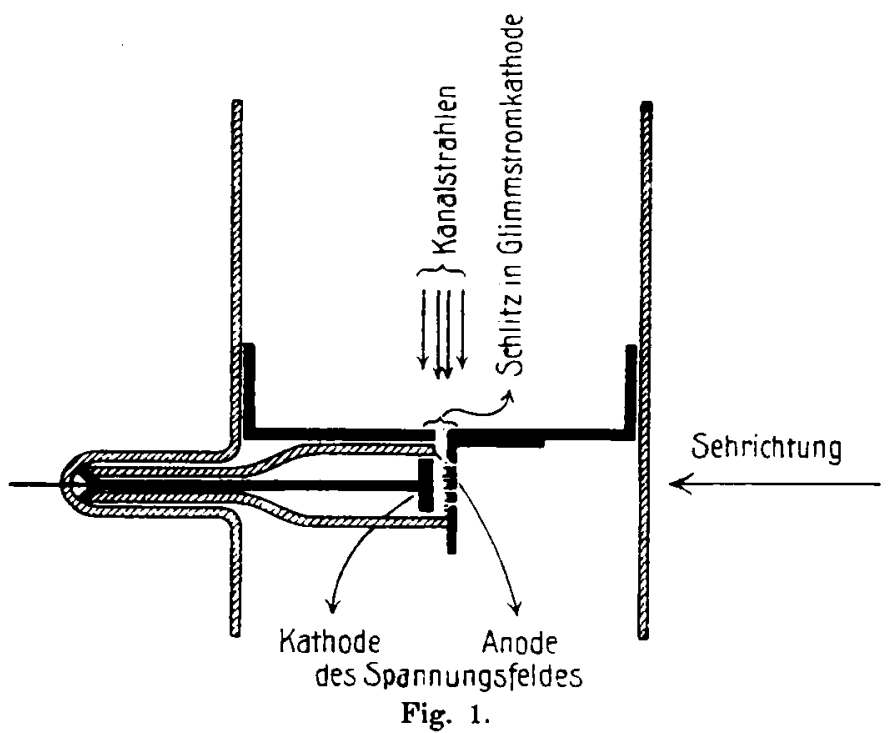

Fig. 1 gibt den axialen Schnitt durch die Glimmstromkathode und das Spannungsfeld. In der Mitte des Bodens der Glimmstromkathode ist ein $3 \mathrm{~mm}$ langer, 1,5 mm breiter Schlitz 
angebracht, durch dieses tritt ein Kanalstrahlenbündel von gleichem UmriB in das Spannungsfeld hinter der Kathode. Auf deren Rückseite ist nämlich die Aluminiumanode des Spannungsfeldes aufgenietet, so daB die Kanalstrahlen gerade an ihrer Oberfläche entlang laufen. Parallel ihr gegenüber in $1,5 \mathrm{~mm}$ Abstand ist als Kathode des Spannungsfeldes eine Aluminiumscheibe von $8 \mathrm{~mm}$ Durchmesser aufgestellt, so daB ebenfalls das Kanalstrahlenbündel an ihrer Oberfläche entlang läuft. Ü̉ber die Aluminiumscheibe ist ein vorne abgeschliffenes Glasrohr so weit geschoben, daB sein vorderer Rand (punktiert) die Anodenfläche berührt. Indes ist in dem Glasrohr unterhalb des Schlitzes in der Glimmstromkathode eine $3 \mathrm{~mm}$ lange, $1, \tilde{5} \mathrm{~mm}$ breite Öffnung gelassen, so $\mathrm{daB}$ das Kanalstrahlenbündel unbehindert in das Spannungsfeld eintreten kann. Es ist sorgfältig darauf geachtet, daB die über die Aluminiumscheibe geschobene Glasröhre diese und die über ihr sitzende Glimmstromkathode nicht berührt, sondern überall $0,5-0,75 \mathrm{~mm}$ Abstand von ihnen hält.

In der Anode des Spannungsfeldes sind dicht nebeneinander drei Reiben $1 \mathrm{~mm}$ weiter Löcher angebracht. Durch diese hindurch, also in der Achse des Spannungsfeldes und senkrecht zum Kanalstrahlenbündel kann der Längseffekt beobachtet werden.

Nun könnte man auf den ersten Blick zu folgendem Einwand geneigt sein. Die in das Spannungsfeld normal zu seiner Achse eintretenden primären positiven Kanalstrahlen werden nach der Kathode des Spannungsfeldes zu beschleunigt und nehmen so eine Geschwindigkeit in der Sehaxe an; das gleiche gilt von den positiven Atomionen, welche durch die StoBwirkung der primären Strahlen im Spannungsfeld geschaffen und nach dessen Kathode zu eine Geschwindigkeit gewinnen. Infolge dieser Geschwindigkeit der primären und sekundären Kanalstrahlen in der Sehachse, kann bei der Beobachtung des Längseffektes des elektrischen Feldes der Dopplereffekt stören.

Die vorstehende Überlegung ist prinzipiell richtig; in quantitativer Hinsicht kann indes dank der Wahl der Versuchsbedingungen der vermutete Dopplereffekt nicht in störender Weise sich bemerkbar machen. Die in der Sehachse von einem einfach geladenen positiven Kanalstrablenion gewonnene Ge- 
schwindigkeit ist nämlich $v=e / m E t$, wo $e / m$ die spez. Ladung des Ions, $E$ die Stärke des Spannungsfeldes, $t$ die Zeitdaner der Beschleunigung ist. Wählt man darum den Weg $l$ der primären Strahlen im Spannungsfeld bis zur Sehachse nur kurz, indem man die Sehachse nahe an die Eintrittsstelle der Kanalstrahlen schiebt und den primären Strahlen eine groBe Geschwindigkeit $v_{0}(6000-10000$ Volt Kathodenfall) gibt, so wird $t=l / v_{0}$ und damit auch $v$ klein. $t=l / v_{0}$ ist zudem die gröBte mögliche Beschleunigungszeit, für den Fall, daB der primäre Kanalstrahl auf dem ganzen Wege $l$ unverändert positiv geladen bleibt. In Wirklichkeit aber sind die meisten primären Kanalstrahlen, wie durch verschiedene Forscher, insbesondere durch W. Wien festgestellt worden ist, bei einem Druck, bei welchem noch Lichtemission statt hat, nur einen Teil der Zeit $t$ ihrer Bewegung durch ein Gas längs eines Weges $l$ positiv geladen. Dieser Umstand bedingt für die meisten primären Kanalstrahlen noch eine weitere Verkleinerung der Geschwindigkeit $v$ in der Sehachse. Zudem haben die primären Strahlen und vor allem die sekundären Kanalstrahlen, die ja im Gesichtsfeld verbleiben, eine störend groBe Geschwindigkeit in der Feldachse erst in unmittelbarer Nähe der Oberfläche die Kathode des Spannungsfeldes gewonnen. Die Lichtemission aus der relativ sehr dünnen Schicht, welche einen störenden Dopplereffekt geben könnte, ist darum unmerklich schwach im Verhältnis zur Lichtemission aus dem übrigen Teil des Spannungsfeldes.

$\mathrm{DaB}$ die vorstehenden Überlegungen für unsere Versuchsbedingungen zutreffend sind, lehrt jedenfalls der Erfolg unserer Beobachtungen. Auf unseren Spektrogrammen ist nämlich nicht einmal bei den dafür besonders empfindlichen Wasserstofflinien auch nur eiue Andeutung eines störenden Dopplereffektes wahrnehmbar.

Nach den obigen Darlegungen ist ohne weiteres ersichtlich, daB man nicht die Kathode, sondern die Anode des Spannungsfeldes mit Löchern für die Beobachtung des Längseffektes zu versehen hat. Es treten nämlich dann nicht die leuchtenden sekundären Kanalstrahlen, sondern nur die im Spannungsfeld erzeugten Kathodenstrahlen durch die Löcher der Anode in den feldlosen Gasraum bis zur Glaswand aus. Diese bringen 
aber ihrer geringen Absorption wegen hier nur eine schwache Lichtemission hervor. Zudem läBt sich diese, wenn sie auftritt, im Spektrogramm von der Lichtemission aus dem Spannungsfeld dank der von uns gewählten optischen Anordnung unterscheiden.

Wie bei der Beobachtung des Quereffektes wurde nämlich auch in der vorliegenden Arbeit das Spannungsfeld scharf auf den Spalt des Spektrographen abgebildet. Wegen der geringen Tiefe des Spannungsfeldes erschienen auch noch die Löcher in seiner Anode scharf in dem Bild auf dem Spalt. Darum waren die Spaltbilder in den Linien des Lichtes aus dem Spannungsfeld ihrer Länge nach nicht kontinuierlich, sondern der Länge nach in kurze Streifchen mit ungeschwärzten $\mathrm{Zwischen-}$ räumen geteilt, wobei die Länge des Streifchens gleich dem Durchmesser eines Lochbildes auf dem Spalt war. Die Linien der Lichtemission infolge der Wirkung der Kathodenstrahlen zwischen den Löchern in der Anode und der Glaswand erschienen dagegen nicht in dieser Weise von lichtleeren Stellen unterbrochen, sondern in ihrer ganzen Länge ausgezeichnet. Dies war insbesondere bei der He-Linie $\lambda$ 5016 Å. der Fall.

Die Abbildung des Spannungsfeldes erfolgte wieder wie früher unter Vorschaltung einer Kalkspat-(Wollaston)-Platte vor das abbildende Objektiv, es wurden also auf dem Spalt unmittelbar übereinander ein rertikal und ein horizontal schwingendes Bild von dem leuchtenden Spannungsfeld entworfen. Waren demnach die in dem Spannungsfeld emittierten Komponenten von zerlegten Serienlinien zum Teil horizontal, zum Teil vertikal geradlinig polarisiert, so fehlten die einen in dem oberen, die anderen in dem unteren Spaltbild. Sezten sich dagegen alle Komponenten in dem oberen Spaltbild geradlinig in das untere Spaltbild fort, so waren die Komponenten entweder unpolarisiert oder zirkular polarisiert. Um wieder zwischen diesen zwei Möglichkeiten zu entscheiden, wurde vor die Kalkspatplatte eine Viertelwellenlängen-Glimmerplatte für Blau so gesetzt, $d a B$ ihre Achsenebene unter $45^{\circ}$ zur Horizontalen lag, daB sie also rechts und links zirkular polarisiertes Licht in vertikal und horizontal geradlinig polarisiertes Licht verwandelte. Setzen sich nunmehr alle Komponenten in dem oberen und unteren Spaltbild geradlinig ineinander fort mit gleicher Intensität, so ist daraus zu folgern, $d a B$ die von dem 
Beobachtungen über den Effeht des elektrischen Feldes usw. II. 989

elektrischen Feld erzwungenen Komponenten einer Serienlinie in der Achse des Feldes unpolarisiert emittiert werden.

An einem Mangel leidet das rorstehende Verfahren zur Untersuchung des Längseffektes, der sich wohl kaum beseitigen lassen wird. Bei der Beobachtung längs der Feldachse ist die Dicke der leuchtenden Schicht nur gering $(1,5 \mathrm{~mm})$ und infolgedessen die Lichtintensität so klein, daB selbst mit dem von uns verwendeten lichtstarken Spektrographen die Expositionszeit auf 3-8 Stunden gesteigert werden muBte.

In Vorbeigehen sei bemerkt, daB die oben beschriebene Anordnung zur Beobachtung des Längseffektes unter Umständen bei entsprechender Verbesserung zur Beobachtung des Quereffektes sich verwenden läBt. Man braucht nämlich die Sehrichtung nur senkrecht zu der Feldachse und zu den Kanalstrahlen zu, stellen.

§ 3. Der Längseffekt im H-Spektrum. Wie in der ersten Abhandlung dargelegt ist, wird die Wasserstofflinie $\mathrm{H}_{\gamma}$ durch ein elektrisches Feld für die Beobachtung quer zu diesem in

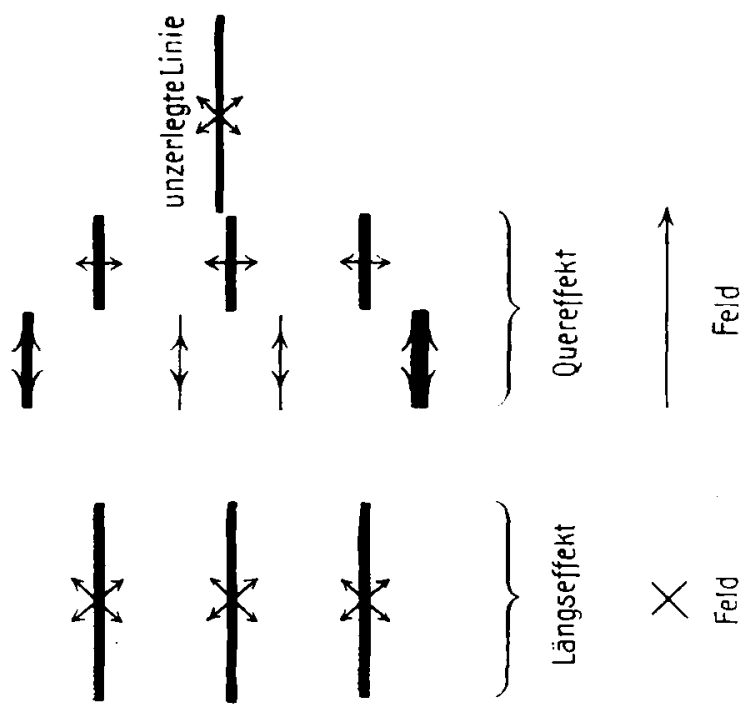

Fig. 2. 
990 J. Stark u. G. Wendt. Beobachtungen über den Effekt useo.

ein Quintett ${ }^{1}$ ) zerlegt, dessen drei innere Komponenten elektrisch senkrecht, dessen zwei äuBere Komponenten parallel zum Feld schwingen. Im Längseffekt erscheinen nun sowohl bei $\mathrm{H}_{\beta}$ wie bei $\mathrm{H}_{y}$ drei unpolarisierte Komponenten, und zwar haben diese dieselbe spektrale Lage (Wellenlänge) wie die drei inneren Komponenten im Quereffekt, welche senkrecht zur Feldachse schwingen. Fig. 2 gibt einen Überblick über die elektrischen Komponenten von $\mathrm{H}_{\gamma}$ und ihre Polarisation für eine Feldstärke von 13000 Volt $\times \mathrm{cm}^{-1}$.

§ 4. Der Längseffekt im He-Spektrum. Wie in der ersten Abhandlung mitgeteilt wurde, werden die Linien der ersten oder diffusen Nebenserien des Heliums unter den angegebenen Versuchsbedingungen in der Quersicht in Komponenten zerlegt, nämlich die Linie $\lambda 4472$ in ein Duplet und $4026 \AA$. in ein Triplet, die Linie $\lambda 4922$ in ein Duplet und $4388 \AA$. in ein Quartett parallel und senkrecht zum Feld schwingender Komponenten. Hierbei fallen die senkrecht mit den parallel schwingenden Komponenten spektral zwar nahezu, aber nicht in allen Fällen genau zusammen.

Der Längseffekt wurde bei den He-Linien $\lambda 4922-4388-$ 4472 Å. spektrographisch beobachtet. Es erschienen bei ihnen allen am Orte der senkrecht zum Feld schwingenden Komponenten im Quereffekt in dem gleichen Intensitätsverbältnis Komponenten, jedoch unpolarisiert wie im Falle des Wasserstoffs.

Aachen, Physik. Instit. d. Techn. Hochschule, 10.Dez. 1913.

1) Durch Anwendung einer größeren Dispersion und eines stärkeren Feldes habe ich unterdes gefunden, dab $\mathrm{H}_{\gamma}$ in einem Feld von 18000 Volt $\times \mathrm{em}^{-1}$ auBer den 2 wei intensiven parallel zum Feld schwingenden Komponenten zwischen ihnen auch zwei weitere parallel dem Feld schwingende Komponenten besitzt, die indes sehr wenig intensiv im Verhältnis zu den äuBeren Komponenten sind. Entsprechend diesem Befund habe ich die Fig. 2 gezeichnet. Die hierbei angewendete Dispersion betrug $1: 8,9 \mathrm{~mm}: \AA$. In einem Feld von 50000 Volt $\times \mathrm{cm}^{-1}$ erschien auch die mittlere senkrecht zum Feld schwingende Komponente in ein Duplet aufgelöst

Stark.

(Eingegangen 10. Februar 1914.) 